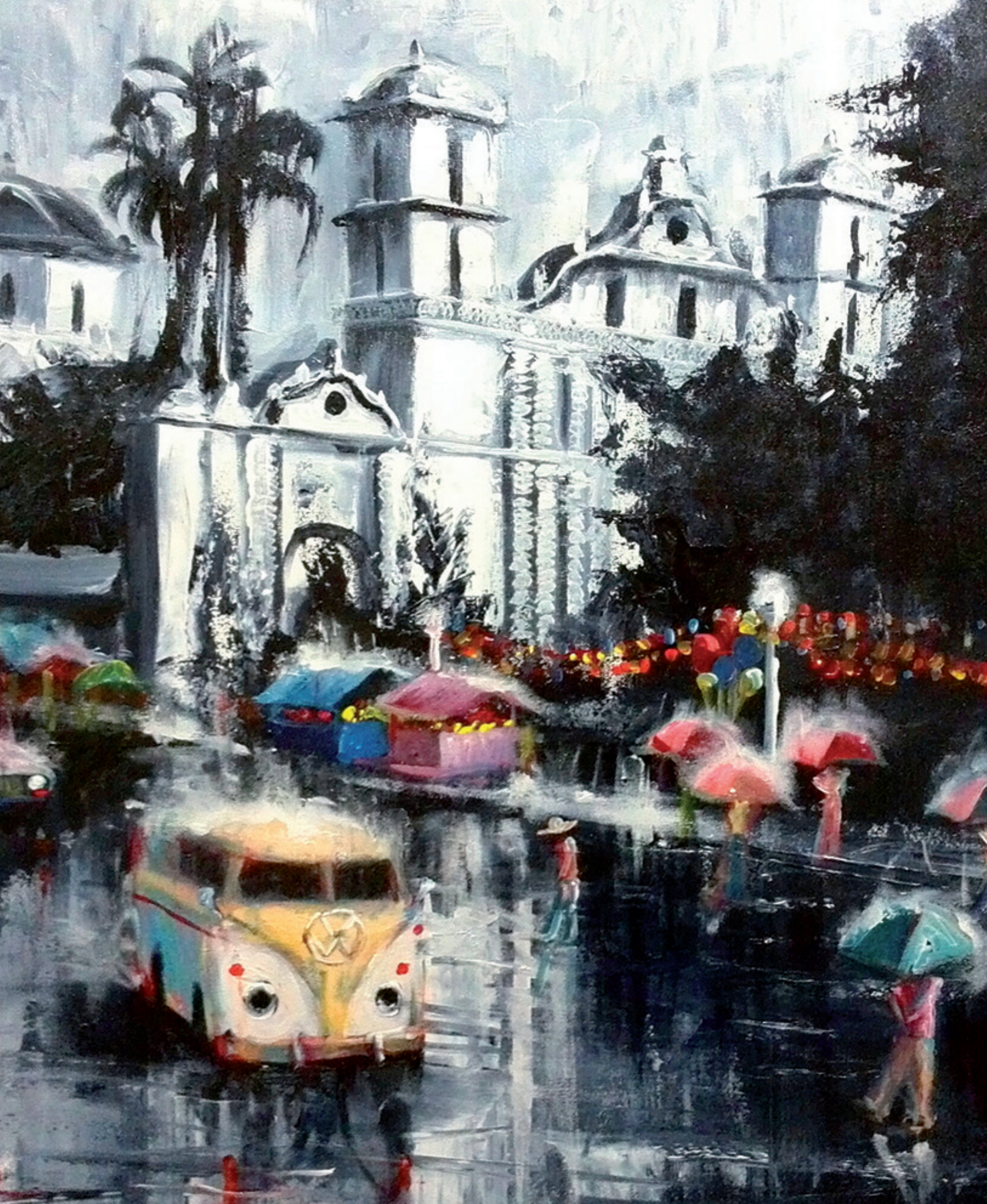




\section{Las mujeres mexicanas en los flujos migratorios laborales hacia Estados Unidos}

OfELIA WOO Morales*

RESUMEN. El escenario demográfico de la migración internacional y específicamente desde México hacia Estados Unidos ha tenido cambios significativos, entre éstos interesa destacar la creciente presencia de las mujeres como migrantes no solo como acompañantes en un proceso de reunificación sino también por su participación en el mercado laboral. En este artículo se presentarán las tendencias del flujo migratorio femenino y masculino respecto a sus características migratorias y laborales a través del tiempo (2000-2012), se recupera la información de la base de datos de la Encuesta sobre Migración en la Frontera Norte de México (EMIF Norte). En el documento se expone de manera sucinta la participación de las mujeres mexicanas en el proceso migratorio como migrantes laborales que conforman parte de un nuevo patrón migratorio diferente al tradicional de reunificación familiar, identificado en la migración de mexicanos hacia Estados Unidos. También se presentan las características de los flujos migratorios del grupo que la encuesta denomina Migrantes Procedentes del Sur que van a Estados Unidos y finalmente se hace algunas reflexiones sobre las diferencias de la migración femenina y masculina en el flujo migratorio en el periodo aludido.

Palabras claves: 1.- migración internacional 2.- flujos laborales 3.- migración laboral femenina 4.- México 5.- Estados Unidos.

SUMMARY. The demographic scenario of the international migration and specifically from Mexico to the United States has had significant changes, notable amongst those is the increasingly presence of women migrants not only as companions in a reunification process but also for their participation in the labor market. This article will present the trends of the female and male migratory flows with respect to their migratory and labor features through time (2000-2012), the information is retrieved from the database of the Encuesta sobre Migración en la Frontera Norte de México (EMIF Norte). The document displays succinctly the participation of the mexican women in the migratory process as labor migrants that conforms part of a new migratory pattern different from the traditional of family reunification, identified in the migration of mexicans to the United States. We also present the migratory flow characteristics of the group which the survey called Migrantes Procedentes del Sur que van a Estados Unidos (Migrants From the South who go to United States) and finally, we make some reflexions about the female and male migration differences in the migratory flow in the afore mentioned period.

Keywords: 1.- International Migration 2.- labor flows 3.- female labor migration 4.-México 5.- United States of America.

\section{Introducción}

El escenario demográfico de la migración internacional y específicamente desde México hacia Estados Unidos ha tenido cambios significativos, entre éstos interesa destacar la creciente presencia de las mujeres como migrantes específicamente en el registro del flujo migratorio.
Las mujeres siempre han estado presentes en la migración mexicana hacia Estados Unidos. Es una emigración de mexicanos hacia el vecino país del norte que inicia desde finales de siglo XIX y continúa hasta la fecha, como señala Massey y Durand (2003), este proceso migratorio es complejo, cambiante y dinámico.

El flujo migratorio ha cambiado en su composición, rutas, lugares de origen y destino. ${ }^{1}$ Para explicar la parti-

Recibido: julio 2014 / Aceptado y versión final agosto 2014.

* Investigadora-Profesora Departamento de Estudios Socio Urbanos, Centro Universitario de Ciencias Sociales y Humanidades, Universidad de Guadalajara.

1. Para una explicación más detallada de nuevas rutas para cruzar Estados Unidos, lugares de origen y destino de migrantes mexicanos se puede revisar la publicación de 2013 de la Encuesta de la Migración en la Frontera Norte de México. Serie anualizada en el capítulo 1.

2. Para Corona (1995) al revisar el levantamiento de la EMIF 1995, señala que "las mujeres tienen una carrera migratoria corta, prácticamente reducida a un viaje, abandonan muy pronto la circularidad" (9). 
cipación de las mujeres en los flujos migratorios Escobar (1999), Trigueros (1999), Corona $(1988,1995)$ hacían referencia que la migración femenina respondía a ciclos migratorios más prolongados a diferencia de la migración masculina $^{2}$, relacionados a la reunificación familiar y a los riesgos que implica para las mujeres realizar un cruce hacia Estados Unidos de manera indocumentada 3 .

Diversos autores (Escobar 1999; Ávila et al 2000), dan cuenta de la participación de hombres y mujeres en el registro de la población migrante, respecto del volumen de migrantes mexicanos en Estados Unidos, las mujeres han representado un porcentaje significativo, más del 45\% desde 1980, en términos relativos no ha aumentado mucho pero en números absolutos la presencia de mujeres es cada vez más notoria. En la Encuesta de la Migración en la Frontera Norte (EMIF) en sus primeras emisiones (1994-1995) sólo el 5.6\% de los flujos registrados eran mujeres, sin embargo para el 2000 llegó al 20\%.

Se ha demostrado con diversas metodologías que la migración femenina es heterogénea Massey y Durand (2003) Durand (1994), Woo (2001), Ávila et al (2000) Arias y Woo 2004, Alonso (2012b) y por su condición de género tiene un patrón migratorio diferente a la migración masculina.

El objetivo de este documento es presentar la participación de las mujeres en los flujos migratorios a través de la Encuesta de Migración de la Frontera Norte de México, en adelante referida EMIF Norte. Se recupera el supuesto de Trigueros que estamos observando una mayor participación de las mujeres en el flujo de migración femenina que tiene un componente laboral y "lo específico de la EMIF es que se enfoca al flujo laboral migratorio" (Boletín CONAPO, 1999:18).

Se presentan las tendencias del flujo migratorio femenino y masculino respecto a sus características migratorias y laborales a través del tiempo de 2000-2012.
En el documento se expone de manera sucinta la participación de las mujeres en el proceso migratorio como migrantes laborales, que conforman parte de un nuevo patrón migratorio diferente del patrón tradicional de reunificación familiar, que Alonso (2012b) denomina patrón emergente, -no necesariamente excluyentes-. Posteriormente, se presentan las características de los flujos migratorios de hombres y mujeres de acuerdo a la EMIF Norte, del grupo que la encuesta denomina Migrantes Procedentes del Sur que van a Estados Unidos y finalmente se hacen algunas reflexiones sobre las diferencias de la migración femenina y masculina en el flujo migratorio.

\section{Nota metodológica sobre la información de la EMIF Norte ${ }^{4}$}

La Encuesta sobre Migración en la Frontera Norte de México, EMIF Norte, contempla un procedimiento de muestreo de poblaciones móviles en localidades fronterizas del norte del país que se realiza desde 1993 a la fecha. A veinte años de su aplicación "La encuesta es única en su tipo en México ya que la metodología y marco muestral aplicados están diseñados para captar personas en movimiento, de tal suerte que puede recabar aspectos más detallados del fenómeno en estudio que no es posible obtener con cuestionarios diseñados para aplicar en hogares" ("Encuesta sobre Migración en la Frontera Norte: 2012:24). Las instituciones que participan en esta Encuesta son: El Colegio de la Frontera Norte, Secretaría de Gobernación, Secretaría de Trabajo y Previsión Social, Secretaría de Relaciones Exteriores.

Las ventajas de esta encuesta son:

- Es una encuesta continua y sistemática de tal manera que se puede podemos observar cambios y continuidades en el desplazamiento de la población.

3. De acuerdo al boletín de CONAPO respecto a la documentación de los y las migrantes con información de la EMIF Norte "las mujeres procuran más que los varones de allegarse algún documento al ingresar a Estados Unidos 55\% pero solo 1 de cada 5 contaba con documentos para trabajar “ (Boletín CONAPO, Año 5, número 13, 2001:5).

4. Quiero agradecer a Orlando García Vega, Jefe del departamento de Estudios de Flujos Migratorios Internacionales de la Secretaría General del Consejo Nacional de Población por apoyarme en el procesamiento de la información estadística de la EMIF Norte. 
- La encuesta se aplica al migrante en el momento del desplazamiento.

- Permite realizar análisis estadísticamente representativo para cada una de las poblaciones objetivo desagregado en dos direcciones: geográfica y temporal.

Las limitaciones que se reconocen son:

- La sobre-enumeración ya que capta desplazamientos no individuos

- Una subestimación de la población femenina porque la encuesta tiene un enfoque laboral.

El objetivo general de la encuesta es "Profundizar en el conocimiento del fenómeno migratorio laboral a la frontera norte de México y a Estados Unidos, destacando sus características, volumen y tendencias, así como sus efectos en el mercado de trabajo e impactos en ambas sociedades" (2013:26).

La población objetivo se han clasificado en los siguientes grupos: migrantes laborales procedentes del sur del país con dirección a Estados Unidos o la frontera norte; procedentes de Estados Unidos y de la frontera norte de México hacia el interior del país (retorno voluntario); los migrantes que son devueltos a México por la Patrulla Fronteriza Estadounidense (retorno forzado).

En este documento se hace referencia al grupo de población que la encuesta identifica migrantes procedentes del sur que emigran a Estados Unidos población mexicana de 15 años $^{5}$ y más ${ }^{6}$. Se recupera la información de los años del 2000 al 2012, de acuerdo a los expertos que han trabajado esta encuesta los años 93, 94, 96, 97 y
98 el levantamiento no se realizó durante todo el año por eso se concentra en el periodo señalado. El periodo que se analiza resulta bastante útil para ver a través del tiempo las variaciones del flujo migratorio de hombres y mujeres y algunas de sus características migratorias y laborales.

Cabe mencionar que en este periodo se presentan dos acontecimientos que marcan efectos importantes en la composición y perfil de los flujos migratorios, el ataque terrorista a Estados Unidos el 11 de septiembre de 2001 y con ello la modificación y aumento de los recursos destinados a vigilar la frontera sur y las reformas de política migratoria generando una política de disuasión a la migración. Segundo, la crisis económica que afectó principalmente la economía estadounidense desde 2008 y con ello el empleo tanto de nativos como de migrantes, llevando al país a una crisis que se compara a la que vivieron en 1929 con la Gran Depresión.

La información que se recupera de los cuestionarios aplicados a la población objetivo es muy vasta y no podríamos en este documento dar cuenta de todos los registros de la base de datos; se seleccionaron algunas variables que permiten identificar por diferencia de sexo: participación en el flujo; motivos para emigrar; ocupación en México; procedencia, edad, escolaridad.

Se presentan en el cuadro 1 los números absolutos de la población referida en este artículo. En las gráficas y cuadros $^{7}$ se registran los porcentajes de estas cantidades por flujo y sexo, de tal manera que se puede observar el comportamiento de las variables en las mujeres y hombres de manera particular, pero también hacer comparaciones cuando sea necesario destacarlo.

5. En el diseño original de la EMIF NORTE, el límite inferior de edad para aplicar la entrevista era de 12 años; sin embargo, dado que este grupo es relativamente pequeño, a partir de 2010 la encuesta se aplica a la población de 15 años o más.

6. De acuerdo al glosario de la EMIF Norte "Los migrantes procedentes del sur. Personas que provienen del sur con respecto a la Frontera Norte de México y se dirigen a Estados Unidos o a una ciudad fronteriza, de 15 años o más, nacidos en México, que llegan a alguna de las ciudades de muestreo y no viven en la localidad de la entrevista o en un país distinto a México, cuyo desplazamiento tiene como motivo trabajar, buscar trabajo, cambiar de residencia u otra razón, siempre y cuando, en este último caso, no tengan fecha comprometida de regreso o trabajo en su lugar de residencia”. (Encuesta de la Migración en la Frontera Norte:2013, 386).

7. Quiero agradecer a Ofelia Medrano por el apoyo en la elaboración de las gráficas y cuadros. 


\section{Cuadro 1. Migrantes procedentes del sur con destino a Estados Unidos}

\begin{tabular}{|r|l|r|r|r|r|r|}
\hline \multirow{2}{*}{ AÑoS } & \multicolumn{3}{|c|}{ Valores Absolutos } & \multicolumn{3}{c|}{ Valores Absolutos } \\
\cline { 2 - 7 } & \multicolumn{1}{|l|}{ Total } & Hombre & Mujeres & Total & Hombre & Mujeres \\
\hline 2000 & 454,707 & 362,325 & 92,383 & 100 & 79.7 & 20.3 \\
\hline 2001 & 405,854 & 303,197 & 102,657 & 100 & 74.7 & 25.3 \\
\hline 2002 & 728,518 & 614,986 & 113,533 & 100 & 84.4 & 15.6 \\
\hline 2003 & 628,175 & 537,094 & 91,081 & 100 & 85.5 & 14.5 \\
\hline 2004 & 542,153 & 482,242 & 59,911 & 100 & 88.9 & 11.1 \\
\hline 2005 & 708,747 & 601,137 & 107,610 & 100 & 84.8 & 15.2 \\
\hline 2006 & 815,569 & 730,495 & 85,074 & 100 & 89.6 & 10.4 \\
\hline 2007 & 855,682 & 750,863 & 104,819 & 100 & 87.8 & 12.2 \\
\hline 2008 & 748,393 & 608,278 & 140,115 & 100 & 81.3 & 18.7 \\
\hline 2009 & 630,449 & 515,685 & 114,764 & 100 & 81.8 & 18.2 \\
\hline 2010 & 492,876 & 365,090 & 127,786 & 100 & 74.1 & 25.9 \\
\hline 2011 & 317,105 & 230,474 & 86,631 & 100 & 72.7 & 27.3 \\
\hline 2012 & 276,145 & 227,976 & 48,169 & 100 & 82.6 & 17.4 \\
\hline
\end{tabular}

Fuente: Elaboración propia Encuesta sobre Migración a la Frontera Norte 2000-2012

\section{Rompiendo estereotipos. La visibilidad de las mujeres en la migración hacia Estados Unidos.}

La producción académica sobre la migración mexicana hacia Estados Unidos es vasta y diversa, pero privilegiada desde una visión androcéntrica desde sus inicios. De tal manera que la participación de las mujeres que se quedaba o emigraban era resultado de la migración masculina y poco habría que dar cuenta.

Para Greco y Boyd (2003) y Pessar $(1999,2007)$ ése carácter asociativo llevó por mucho tiempo a la invisibilidad de las mujeres en los estudios de la migración. El pionero en registrar desde México y dar cuenta de la participación de las mujeres en la migración hacia Estados Unidos como parte del proceso de reunificación familiar desde principios del siglo XX fue Gamio (1930), sin embargo, tuvieron que pasar muchas décadas para que la mujeres aparecieran nuevamente en el escenario de la migración hacia Estados Unidos, es hasta la década de los ochenta con Bustamante (1988) que da cuenta de la presencia de las mujeres en los flujos migratorios y Durand (1994), señalaba en el periodo de la migración indocumentada (1964-1986), "se modificó paulatinamente el patrón migratorio de hombres solos, promovido por le época de braceros, la migración femenina rebasó con mucho la proporción de épocas anteriores (1994:135). Aunque hubo el reconocimiento de una mayor participación de la mujer en la migración poca atención se daba como actor social.

En esta década de los noventa hasta la fecha, se iniciaría una generación de nuevos estudios de académicos mexicanos que centran la atención a la migración de las mujeres. Se realizaron estudios importantes con metodologías cualitativas y cuantitativas que exponen la experiencia de las mujeres mexicanas al emigrar, documentan quienes son, por qué emigran, hacia dónde se dirigen, que diferencias existe con los hombres migrantes. (Avila et. al. 2001, CONAPO 2000, Woo 2001, Arias y Woo 2004, Alonso (2012b), Berumen y Santiago, (2012), así también se realizan artículos que presentan los riesgos a los que se enfrentan las mujeres al realizar una emigración sin documentos, cruzando la frontera norte de México hacia Estados Unidos (Woo 2004), Marroni (2005), Alonso (2012a).

Ávila et. al. (2001) hace referencia a la participación de las mujeres en la migración temporal antes de los noventa con registros de la Encuesta de la Dinámica Demográfica (ENADID) demostrando que no es un fenómeno reciente. Sin embargo, se puede considerar un hito la participación femenina y registro de la misma después que fue aprobada la Ley de Inmigración de 1986 Immigration Reform and Control Act (IRCA) mejor conocida como Simpson-Rodino.

Del total de solicitudes de legalización amparadas en la Ley Simpson Rodino en los programas "Normal" (Legaly Autorizad Workers) y de trabajadores agrícolas especiales (Special Agricultural Workers) 43 y 17 por ciento era mujeres (Bean et al 1989).

Las estadísticas sobre migración -específicamente sobre las mujeres- difieren porque son captadas con metodología y conceptos diferentes, como señala López Villar (2002), las distintas categorías de migrantes y cuestiones conceptuales no posibilita recolectar un total 
de los mismos. De ahí que la visibilidad de las mujeres en la migración hacia Estados Unidos depende de la metodología empleada y la población estudiada, es diferente cuando se hace referencia al stock de población y cuando habla de flujos migratorios.

\subsection{La migración femenina, migración laboral}

De acuerdo a las estadísticas del Instituto Nacional de Estadísticas Geografía e Informática (INEGI), México contaba con una población de 112,336, 538 personas para el 2010. Berumen y Santiago mencionan que para el mismo año existía aproximadamente 12 millones de inmigrantes mexicanos (2012:233), el 46.5 por ciento mujeres (ibídem). Para los autores la tasa de crecimiento estimada en 1980 era 11.8 y para el 2010 fue de 2.3 en el caso de los migrantes varones, con respecto a las mujeres fue en 1980 de 10.6 y para el 2010 fue de 3.1. Los autores hacen una crítica al registro de la migración masculina en los censos de Estados Unidos. ${ }^{8}$

Berumen y Santiago plantean que es difícil precisar si existe una feminización o masculinización cuantitativa, pero reconocen la necesidad de analizar la participación de la mujer con mayor detalle, considerando el contexto histórico y el carácter temporal/estacional del patrón migratorio que prevaleció el siglo pasado y el patrón de migración de mayor pertenencia al que transitó (2012:233).

Coincidimos con los autores, la migración de mexicanos hacia Estados Unidos ha presentado cambios importantes en el patrón migratorio y el perfil del migrante de tal manera que es difícil precisar estadísticamente sí existe una feminización cuantitativa de la migración, sin embargo, lo que es evidente es una feminización cualitativa ${ }^{9}$, que refleja un cambio en el patrón y perfil del migrante y la participación de la mujer en la migración y en el mercado laboral en Estados Unidos ha estado sub-representada en los diferentes periodos que ha tenido el proceso migratorio.

Como se decía al inicio del artículo, la migración desde México hacia Estados Unidos, es de larga data. En un principio estuvo caracterizada por la inestabilidad política de México (Revolución de 1910 y la Guerra de los Cristeros 1930) y posteriormente por la demanda laboral de Estados Unidos cuando participa en la segunda guerra mundial y necesita mano de obra para trabajar, principalmente en el sector agrícola y la construcción de las vías férreas, Durand (1994) hace referencia a diferentes etapas de la migración: la del enganche, la deportaciones, del programa bracero, la de los indocumentados y los rodinos. Lo que interesa destacar de estas etapas es que era una migración de carácter laboral, predominantemente masculina, provenía del sector agrícola y era temporal.

Diversos autores mencionan que IRCA no sólo cambio el perfil demográfico de los migrantes, al observar una mayor presencia de mujeres y familias, sino también, cambió el patrón migratorio de ser temporal, circular, a uno de mayor permanencia en Estados Unidos, provocado por el proceso de reunificación familiar, pero también por el aumento en la vigilancia de la frontera sur de Estados Unidos para detener y contener el flujo migratorio.

De manera paradójica, las políticas migratorias de disuasión a la migración -principalmente indocumentadano contuvo ni detuvo, al contrario se observa un mayor crecimiento desde 1980, algunos autores como SassenKoob y Smith (1992) y Portes (1995) lo atribuyen a la reestructuración de la economía estadounidense, señalaban que las principales ocupaciones de la población migrante estaban relacionadas a este proceso de reestructuración

8. Ver cuadro 7.1 Población nacida en México con residencia en Estados Unidos, según sexo, 1970-2010, página 233 en Berumen Sandoval Salvador y Julio Santiago Hernández (2012), "Las mujeres en el proceso migratorio México-Estados Unidos. ¿Hacia una feminización de la migración?, en Migración y familia. Una mirada más humana para el estudio de la migración internacional. Salvador Berumen, Nina Frías y Julio Santiago (coordinadores) México, SPMAR-UPMCEM, INM Colección Migración.

9. Podemos encontrar una discusión muy importante sobre el registro de la migración femenina y la feminización cuantitativa y cualitativa de las migraciones en América Latina en el documento de Pizarro Martínez Jorge (2003). El mapa migratorio de América Latina y el Caribe, las mujeres y el género, Serie Población y Desarrollo, Santiago de Chile, CEPAL. ttp://www.catedradh.unesco.unam.mx/BibliotecaV2/Documentos/Trata/Libros/mapa_genero.pdf consultado 10 de abril de 2010. 
en el que prevalecen trabajos de subcontratación, flexibles, poco calificados y de bajos salarios, y la convivencia con trabajos altamente calificados que demandan trabajadores de baja calificación para ocuparlos principalmente en el sector servicios.

\subsection{Migración familiar vs migración laboral}

Las mujeres mexicanas en el flujo migratorio laboral hacia Estados Unidos.

Como se puede observar en los registros de 2000 . 2012, en la gráfica 1 en el flujo de migrantes procedentes del sur hacia Estados Unidos existen variaciones significativas en el porcentaje de la participación de las mujeres ${ }^{10}$ a diferencia de los hombres que es más constante. Existe un comportamiento inverso del porcentaje de los hombres con respecto a las mujeres a partir de 2008 cae la participación de los hombres y el de las mujeres aumenta. Lo anterior se puede explicar por el patrón migratorio de los hombres que se vio afectado por la crisis económica de Estados Unidos, en cambio la migración de las mujeres al ser multicausal no se observa este efecto, además, de que se ha documentado que la participación laboral de las mujeres no se redujo tan drásticamente como la de los hombres con la crisis aludida.

\section{Gráfico 1. Migrantes procedentes del sur hacia Estados Unidos, hombres y mujeres}

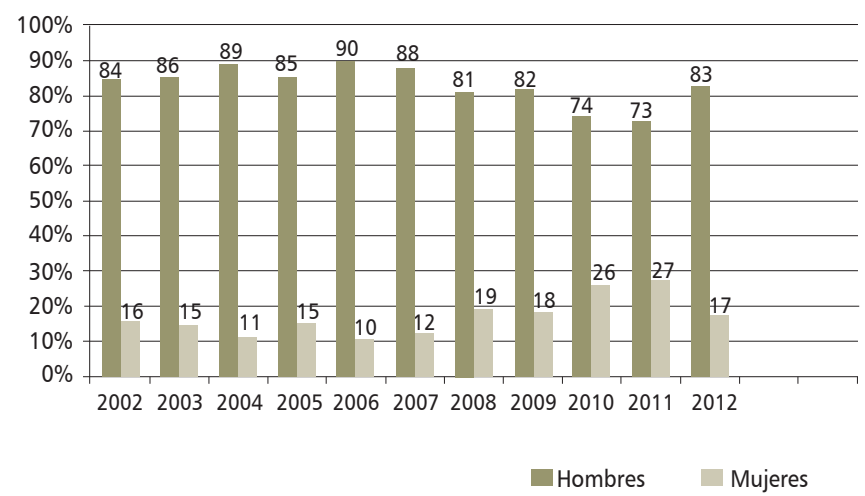

Fuente: elaboración propia. Encuesta sobre Migración en la Frontera Norte de México 2000-2012
Como se señala en el reporte de la EMIF en 2010 “- Aunque históricamente la participación femenina en el flujo migratorio internacional ha sido baja en comparación con los hombres, cada vez son más las mujeres que llegan a la frontera norte con la intención de llegar a Estados Unidos para trabajar o buscar trabajo y mejorar sus condiciones de vida. Asimismo, en algunas ciudades fronterizas se ha observado una mayor presencia de niños, niñas y adolescentes (NNA) migrantes, quienes no sólo migran por motivos de acompañamiento y reunificación familiar, sino también con objetivos laborales" $(2012: 16)^{11}$. Se menciona esta referencia porque es importante reconocer que existe la incorporación a estos flujos migratorios de otras poblaciones como niños, niñas y adolescentes que no se está registrando y que la EMIF deja de lado toda población menor de 15 años.

La emigración de familias y otros grupos etarios (NNA) se puede relacionar con el incremento en la pobreza en el país, desde 2000 a la fecha (2014) pasó de $41 . \%$ a $52 \%$, como resultado del modelo neoliberal que provocó la retirada del Estado en la provisión de servicios de salud (educación, salud, etc). "Se estableció una forma de política pública que introdujo una fragmentación y exclusión permanente de grandes sectores de la sociedad...) Damian (2012:295-296).

\subsection{Motivos de migración}

La migración de los mexicanos hacia Estados Unidos ha estado vinculada históricamente a la oferta y demanda de mano de obra además de las condiciones estructurales que mencionamos párrafos arriba. De ahí que los acercamientos teóricos para explicarla predominaba la teoría neoclásica que privilegiaba las diferencias salariales, condiciones de empleo y costos de migración como una decisión personal para maximizar el ingreso, o como la Nueva Economía de la Migración que analiza las condiciones de mercados diversos (mercados laborales,

10. En los registros de la EMIF en la década de los noventa era tan baja (5.3\% en 1993) que creemos que los doce años que analizamos nos dan cuenta de un aumento significativo de la participación de las mujeres en el flujo migratorio.

11. En el reporte de la EMIF 2010 hace referencia de que se trata de un flujo principalmente de indocumentados. 
mercados de futuro, mercados de capital, mercados de seguro), para reducir riesgos en el ingreso familiar; hasta las teorías que conceptualizan los procesos causales a diferentes niveles de análisis (individual, familiar, nacional e internacional) como la Teoría de Mercados Duales en donde la migración está vinculada a los requerimientos estructurales de las economías.

Estos modelos teóricos dejaban de lado la migración femenina y su participación en los flujos migratorios y en el mercado laboral en el vecino país, ya que se aludía que las mujeres emigraban solo por reunificación familiar, sin embargo, la perspectiva de género nos ha permitido explicar que la migración de las mujeres tiene un patrón migratorio diferente respecto al de los hombres, son actores sociales que emigran por diversos factores, no necesariamente excluyentes, que responde a su condición de género, curso y ciclo de vida individual y familiar tales como: reunificación familiar, buscar trabajo, refugio, asilo, mejores condiciones económicas, laborales, profesionales, mayor independencia familiar, hasta huir de la violencia doméstica. Pessar (1999 y 2007), Hondagneu-Sotelo (1994), Woo (1995 y 2007).
Como se observa en el cuadro 2, respecto a los motivos para emigrar, en el caso de los hombres es contundente para trabajar o buscar trabajo, en cambio en el caso de las mujeres se diversifican los motivos, pero hay un aumento significativo para buscar trabajo, aunque predomine la reunificación familiar como muestra el cuadro 3. Diversos estudios con metodología cualitativa dan cuenta que las mujeres se incorporan al mercado laboral en Estados Unidos independientemente de los motivos de emigración, porque como dicen ellas "si no trabajas no comes". También es importante considerar que este patrón de emigración femenino llamado "emergente" por Alonso ya se había identificado desde la década de los ochenta, por lo que se puede recuperar de esta denominación es que efectivamente nos ayuda a diferenciar del que llama el patrón "tradicional", al de reunificación familiar.

\subsection{Condición laboral en México}

México así como los países de América Latina ha tenido importantes transformaciones en su economía, el modelo neoliberal aplicado en nuestro país desde 1983 a

Cuadro 2. Motivo de los hombres para cruzar la frontera de Estados Unidos

\begin{tabular}{|c|c|c|c|c|c|c|c|c|c|c|c|c|c|}
\hline Motivos para cruzar a Estados Unidos & 2000 & 2001 & 2002 & 2003 & 2004 & 2005 & 2006 & 2007 & 2008 & 2009 & 2010 & 2011 & 2012 \\
\hline Trabajar o buscar trabajo & 71.7 & 68.4 & 82.3 & 86.2 & 89.0 & 86.2 & 88.7 & 85.2 & 80.9 & 82.4 & 81.0 & 78.1 & 82.0 \\
\hline Reunirse con familiares o amigos & 14.9 & 14.3 & 8.5 & 6.8 & 7.5 & 9.8 & 8.5 & 11.2 & 12.5 & 12.3 & 14.3 & 18.6 & 11.6 \\
\hline Paseo, negocio, compras o estudiar & 12.2 & 16.4 & 8.9 & 6.5 & 3.4 & 3.7 & 2.7 & 3.4 & 6.5 & 5.1 & 3.3 & 2.3 & 3.9 \\
\hline Para quedarse a vivir allá * & - & - & - & - & - & - & - & - & - & - & 0.5 & 1.0 & 2.4 \\
\hline Otro & 1.2 & 0.9 & 0.4 & 0.5 & 0.1 & 0.3 & 0.0 & 0.1 & 0.1 & 0.2 & 0.9 & 0.1 & 0.1 \\
\hline
\end{tabular}

Fuente: elaboración propia. Encuesta sobre la migración a la Frontera Norte de México, 2000-2012

*Nota: quedarse a vivir allá se refiere quedarse a vivir en Estados Unidos

Cuadro 3. Motivo de las mujeres para cruzar la frontera de Estados Unidos

\begin{tabular}{|c|c|c|c|c|c|c|c|c|c|c|c|c|c|}
\hline Motivos para cruzar a Estados Unidos & 2000 & 2001 & 2002 & 2003 & 2004 & 2005 & 2006 & 2007 & 2008 & 2009 & 2010 & 2011 & 2012 \\
\hline Reunirse con familiares o amigos & 56.5 & 47.4 & 25.7 & 48.2 & 42.0 & 42.0 & 46.2 & 47.7 & 48.0 & 63.5 & 76.3 & 76.9 & 52.7 \\
\hline Paseo, negocio, compras o estudiar & 24.8 & 34.2 & 43.6 & 21.1 & 11.8 & 10.6 & 14.1 & 13.3 & 28.7 & 14.9 & 8.3 & 11.1 & 16.3 \\
\hline Trabajar o buscar trabajo & 17.7 & 15.9 & 26.8 & 25.9 & 45.5 & 46.9 & 37.6 & 37.7 & 22.1 & 21.2 & 13.0 & 9.8 & 20.7 \\
\hline Para quedarse a vivir allá * & - & - & - & - & - & - & - & - & - & - & 1.6 & 2.2 & 10.0 \\
\hline Otro & 1.0 & 2.6 & 3.9 & 4.8 & 0.7 & 0.6 & 2.1 & 1.3 & 1.2 & 0.4 & 0.9 & 0.0 & 0.3 \\
\hline
\end{tabular}

Fuente: elaboración propia. Encuesta sobre la migración a la Frontera Norte de México, 2000-2012

* Nota: quedarse a vivir allá se refiere quedarse a vivir en Estados Unidos 
la fecha ha dejado profundas crisis económicas y sociales. Calva y Salazar señalan que en México durante el periodo de 1953 y 1982 con el modelo precedente al neoliberal, el Producto Interno Bruto (PIB) creció a 6.3\% anual y el empleo a una tasa media de 9.7. En cambio de 1983 a 2010 con el modelo neoliberal el PIB creció a una tasa media de 2.2 y el empleo formal 2.7 anual (2012:23). Los autores no sólo demuestran el fracaso y el deterioro de la economía y del empleo sino también la precariedad del mismo.

De acuerdo a los datos del Instituto Nacional de Geografía (INEGI), la población ocupada por sector de actividad económica para 2012 fue: $13.6 \%$ para el sector agrícola, $23.3 \%$ al sector industrial, $62.4 \%$ al sector servicios ${ }^{12}$. Es importante mencionar que en la misma fuente se registra una disminución significativa en 1995 para el sector agrícola que contaba con 23.7 $\%$ de población ocupada, un crecimiento del sector industrial pues contaba con $53 \%$ y sin variaciones en el sector industrial.

Cabe señalar que la participación económica de las mujeres tuvo un aumento significativo de 1995 a 2012, de $36.8 \%$ a $43.5 \%$ y una disminución en el caso de los hombres para el mismo período de $80.9 \%$ a $77.5 \%$. Lo anterior es necesario tomarlo con cautela no significa que las mujeres estén en ventaja respecto a los hombres, lo que se ha demostrado por varios estudiosos del tema es que las mujeres se incorporan en actividades más precarias principalmente en el sector servicios.

La participación de las mujeres en el mercado laboral tanto en México como en otras latitudes principalmente en América Latina es un tema ampliamente estudiado considerado como un cambio significativo en la dinámica de las familias y de las mujeres.

Se hace referencia que existe un cambio importante en la participación económica ya que predominaban las mujeres jóvenes y solteras las que se incorporaban y permanecían en el mercado laboral, actualmente las mujeres

Grafico 2. Condición laboral de hombres y mujeres migrantes hacia Estados Unidos 2000-2012

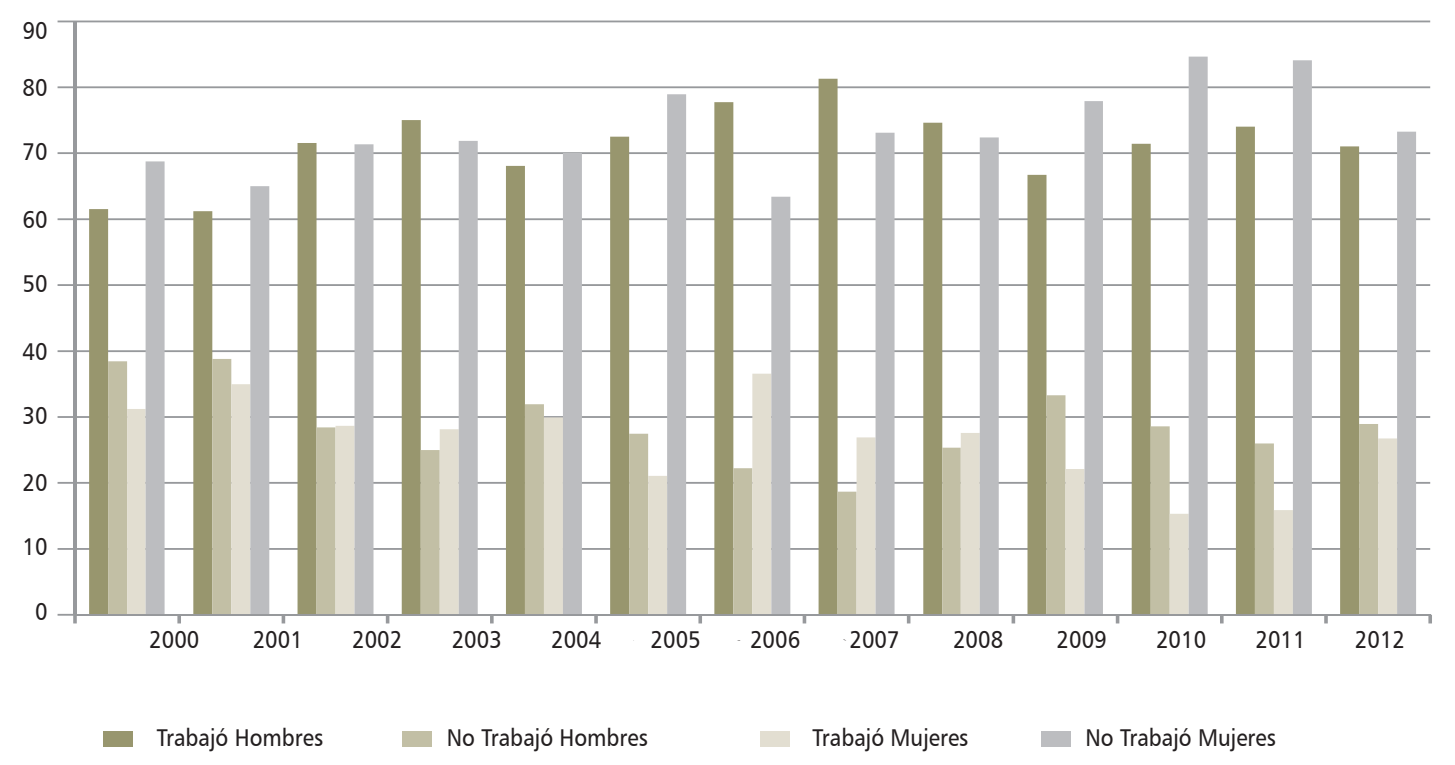

Fuente: Elaboración propia, Encuesta sobre migración en la Frontera Norte de México, 2000-2012

12. El sector agrícola comprende: agricultura, ganadería, silvicultura y pesca; el industrial, industrias extractivas, manufactureras, de electricidad y construcción; el de servicios, comprende restaurantes y servicios de alojamiento, comunicaciones y transportes, correo y almacenamiento, servicios financieros y otros servicios, gobierno y organismos internacionales, fuente: México de un vistazo 2012, Instituto Nacional de Geografía, 2012 consultado en http://www.inegi.org. mx/prod_serv/contenidos/espanol/bvinegi/productos/integracion/pais/mexvista/2012/Mex_vi12.pdf 28 de julio de 2014. 
permanecen en su etapa reproductiva y se registra la participación más elevada en las mujeres de 30 y 40 años. ${ }^{13}$

En el caso de las mujeres que emigran esta condición no parece muy elevada, ya que de las migrantes que declararon que trabajaba antes de emigrar oscila entre el $20 \mathrm{y}$ $30 \%$, muy bajo en relación a las mujeres que trabajan en México y muy por debajo el porcentaje en relación con los hombres migrantes como se puede observar en la gráfica 2 más del 60\% de los hombres trabajó antes de emigrar.

De los hombres que trabajaban, lo hacían principalmente en agricultura e industria, cabe destacar que en el 2000, 2001 y 2002 responde en mucho a la relación de oferta y demanda de mano laboral en Estados Unidos, cabe recordar que más del 80\% de los trabajadores en la agricultura en el vecino país son mexicanos (Ver cuadro 4). En cambio en las mujeres el porcentaje de profesionistas, técnicos, administrativos es superior a cualquier otro oficio declarado, a excepción de 2004,
2005 y 2009 que predomina comercio, le sigue trabajadores en servicios personales y públicos (Ver cuadro 5), a diferencia de los hombres la participación de profesionistas y posteriormente sobresale, en 2000, 2001 y 2002, después disminuye considerablemente. Como parte de la transformación de los mercados laborales se hace referencia a la tendencia de emigración de población altamente calificada y México forma parte de la misma, aunque no necesariamente la población que aludimos, ya que, es una población que tiene un nivel de escolaridad elevada que lamentablemente se incorpora a trabajos precarios, flexibles y no necesariamente en la formación profesional adquirida.

\subsection{Procedencia de migrantes}

Una de las características que diferenciaba al flujo de migrantes en la década de los ochenta era que había aumentado la procedencia de localidades urbana respecto

Cuadro 4. Oficio desempeñado en México por hombres migrantes 2000-2012

\begin{tabular}{|c|c|c|c|c|c|c|c|c|c|c|c|c|c|}
\hline Oficios desempeñados & 2000 & 2001 & 2002 & 2003 & 2004 & 2005 & 2006 & 2007 & 2008 & 2009 & 2010 & 2011 & 2012 \\
\hline Trabajadores agropecuarios & 39.8 & 41.3 & 52.8 & 51.1 & 45.4 & 47.6 & 52.3 & 50.1 & 42.6 & 42.3 & 48.5 & 48.0 & 50.3 \\
\hline Trabajadores industriales & 28.8 & 22.8 & 19.9 & 28.0 & 32.1 & 32.2 & 31.3 & 32.4 & 33.6 & 36.2 & 32.1 & 33.2 & 31.4 \\
\hline $\begin{array}{l}\text { Profesionistas, técnicos y } \\
\text { personal administrativo }\end{array}$ & 11.7 & 19.5 & 15.3 & 6.8 & 6.6 & 5.2 & 3.7 & 5.2 & 7.1 & 5.3 & 3.4 & 3.5 & 4.7 \\
\hline Comerciantes & 8.4 & 6.6 & 6.5 & 7.4 & 7.3 & 7.9 & 5.4 & 5.8 & 8.5 & 8.6 & 6.9 & 6.3 & 5.6 \\
\hline $\begin{array}{l}\text { Trabajadores en servicios } \\
\text { personales y públicos }\end{array}$ & 11.2 & 9.7 & 5.4 & 6.8 & 8.7 & 7.1 & 7.4 & 6.6 & 8.1 & 7.6 & 9.1 & 9.0 & 8.0 \\
\hline Total & 100.0 & 100.0 & 100.0 & 100.0 & 100.0 & 100.0 & 100.0 & 100.0 & 100.0 & 100.0 & 100.0 & 100.0 & 100.0 \\
\hline
\end{tabular}

Fuente: Elaboración Propia. Encuesta sobre migración en la Frontera Norte de México, 2000-2012

Cuadro 5. Oficio en desempeñado en México por mujeres 2000-2012

\begin{tabular}{|c|c|c|c|c|c|c|c|c|c|c|c|c|c|}
\hline Oficios desempeñados & 2000 & 2001 & 2002 & 2003 & 2004 & 2005 & 2006 & 2007 & 2008 & 2009 & 2010 & 2011 & 2012 \\
\hline Trabajadores agropecuarios & 5.7 & 12.7 & 10.7 & 12.8 & 16.6 & 6.1 & 5.4 & 9.8 & 9.8 & 7.4 & 9.0 & 9.3 & 7.1 \\
\hline Trabajadores industriales & 15.7 & 9.6 & 8.2 & 14.1 & 8.6 & 8.1 & 14.8 & 20.1 & 16.4 & 13.8 & 20.7 & 7.6 & 5.7 \\
\hline $\begin{array}{l}\text { Profesionistas, técnicos y } \\
\text { personal administrativo }\end{array}$ & 37.5 & 34.6 & 41.6 & 31.2 & 9.1 & 25.4 & 28.0 & 28.9 & 32.0 & 26.8 & 18.6 & 56.0 & 53.4 \\
\hline Comerciantes & 19.3 & 23.5 & 19.9 & 31.6 & 42.4 & 43.6 & 25.7 & 11.8 & 25.9 & 32.0 & 19.8 & 12.5 & 19.2 \\
\hline $\begin{array}{l}\text { Trabajadores en servicios } \\
\text { personales y públicos }\end{array}$ & 21.8 & 19.6 & 19.5 & 10.3 & 23.3 & 16.9 & 26.1 & 29.4 & 15.9 & 20.0 & 31.9 & 14.7 & 14.5 \\
\hline Total & 100.0 & 100.0 & 100.0 & 100.0 & 100.0 & 100.0 & 100.0 & 100.0 & 100.0 & 100.0 & 100.0 & 100.0 & 100.0 \\
\hline
\end{tabular}

Fuente: Elaboración Propia. Encuesta sobre migración en la Frontera Norte de México, 2000-2012

13. Fuente: Sistema de indicadores de género del Instituto Nacional de las Mujeres obtenido de la Encuesta Nacional de Ocupación. 
a las rurales (Bustamante 1988, Corona 1988, Massey y Durand 2003; Durand 1994); lo anterior se podía explicar por la tendencia nacional al convertirse México en un país con población más urbana desde la década de los setenta, por el desplazamiento de la población de las áreas rurales a las ciudades por el proceso de modernización que llevó a la crisis del sector agrícola en nuestro país y el crecimiento de las ciudades.

En el caso de la población que participa en los flujos migratorios se supone que las ocupaciones de las y los migrantes pueden estar ampliamente relacionadas con los lugares de procedencia. De acuerdo a la EMIF Norte destaca el porcentaje de las mujeres que provienen de localidades urbanas ${ }^{14}$ a diferencia de los hombres (Gráfico 3). Aunque no hay que desdeñar el aumento significativo que se observa en el 2004, 2005, 2007 y 2012 para el caso de los hombres de localidades no urbanas y en el 2004 y 2005 en los mujeres. Diversos estudios nos dan cuenta de la incorporación de Estados de emigración emergente como Veracruz y Chiapas que están expulsando población de áreas rurales que han sido afectadas por la crisis del sector agropecuario en México.

\section{- Edad}

Avila et al (2000) hacían referencia a una migración masculina joven, el 40\% oscilaba de 15 a 25 años, en la serie histórica que se muestra de la EMIF Norte se encuentra un aumento significativo de migrantes varones que tiene un promedio de edad de 30 a 35 años, sin embargo en el caso las mujeres es desde los 35 a los 50 principalmente en 2010 y 2011 (ver cuadro 6), existen
Gráfico 3. Tipo de localidad de hombres y mujeres migrantes 2000-2012

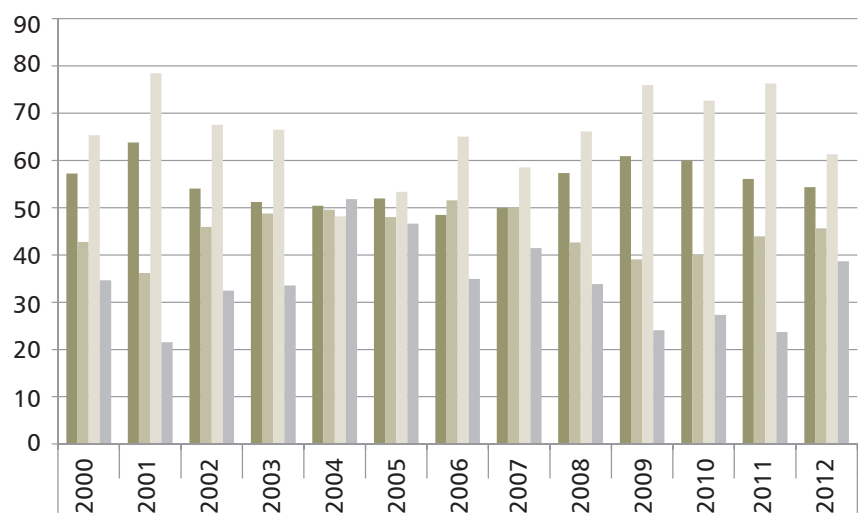

-Urbana Hombres No Urbana Hombres Urbana Mujeres No Urbana Mujeres

Fuente: Elaboración propia: Encuesta sobre migración en la Frontera Norte de México, 2000-20012

varias hipótesis, la falta de oportunidades laborales para hombres de 35 y 40 años y más, y en el caso de las mujeres porque responden a diversos ciclos de vida, en el que las mujeres están emigrando no para reunirse con su marido, sino para reunirse con sus hijos; otra hipótesis, es que la falta de oportunidades obliga a las mujeres en edades avanzadas buscar mejores oportunidades de vida en Estados Unidos.

\section{- Escolaridad}

Uno de los pocos logros se puede reconocer es el avance en el acceso de la educación para las mujeres en México, de tal manera que se redujo la brecha de género en matricula del Sistema de Educación Pública, de ahí que cuando autores como Corona (1988) y Bustamante

Cuadro 6. Edad promedio hombres y mujeres migrantes $2000-2012$
\begin{tabular}{|l|r|r|r|r|r|r|r|r|r|r|r|r|r|}
\hline Edad & 2000 & 2001 & 2002 & 2003 & 2004 & 2005 & 2006 & 2007 & 2008 & 2009 & 2010 & 2011 & 2012 \\
\hline Hombres & 35.2 & 36.2 & 35.2 & 32.3 & 31.1 & 29.6 & 30.1 & 30.3 & 32.4 & 33.5 & 35.4 & 35.1 & 32.6 \\
\hline Mujeres & 40.4 & 39.9 & 34.5 & 32.5 & 34.9 & 34.9 & 38.2 & 38.5 & 40.3 & 42.3 & 50.8 & 50.5 & 41.6 \\
\hline total & 100 & 100 & 100 & 100 & 100 & 100 & 100 & 100 & 100 & 100 & 100 & 100 & 100 \\
\hline
\end{tabular}

Fuente: Elaboración propia. Encuesta migración en la Frontera Norte de México, 2000-2012 
(1988), identificaron desde la década de los ochenta que las mujeres tenían un nivel más elevado de escolaridad respecto al de los hombres se aludía que respondía a la tendencia nacional.

Sin embargo, es interesante observar actualmente que la escolaridad de las mujeres sigue en niveles escolares más elevados sino que en bachillerato completo y licenciatura los porcentajes casi se duplican, en la serie histórica en 2012, el 23.8\% de las mujeres tenía licenciatura y sólo el 4.6\% los hombres (ver gráfica 4).

Si relacionamos estos datos con el oficio de las migrantes y los migrantes se puede inferir que existe una fuga de cerebros, que independientemente de su incorporación en el mercado laboral, México está subsidiando mano de obra barata pero sobre calificada.

\section{Algunas reflexiones}

México es un país expulsor de emigrantes y el principal lugar de destino ha sido históricamente Estados Unidos. El inicio del nuevo siglo y milenio parece indicar que la migración no se detendrá a pesar de las crisis económicas internacionales, ni cambiará en corto plazo el modelo neoliberal que ha llevado al deterioro de la calidad de vida de la población.

Los flujos migratorios se deben entender y explicar en los contexto de origen, tránsito y destino de la población migrante pero también de los proyectos de vida individual y familiar, no fue el objetivo de este artículo pero se reconococe la necesidad de tenerlo presente. El objetivo fue más modesto, presentar la migración de las mujeres mexicanas en el flujo migratorio hacia Estados Unidos con un patrón de migración laboral.

Existe una transformación de los mercados laborales internacionales que hace necesaria la mano de obra migrante, excedente, precaria y desechable, que responda a los ciclos económicos de los países desarrollados.

Las mujeres como los hombres, emigran para buscar trabajo en Estados Unidos, se parte del supuesto, que las mujeres que declaran motivos familiares saben que en algún momento tienen que buscar trabajo, porque vivir en Estados Unidos implica gastar dólares, por lo que se necesita ganar dólares. Con la crisis del 2008 se demostró que la principal población migrante afectada fue la masculina, ya que participa más en los sectores de la economía que tuvieron importantes caídas, como la construcción.

Gráfico 4. Escolaridad de hombres y mujeres migrantes 2000-2012

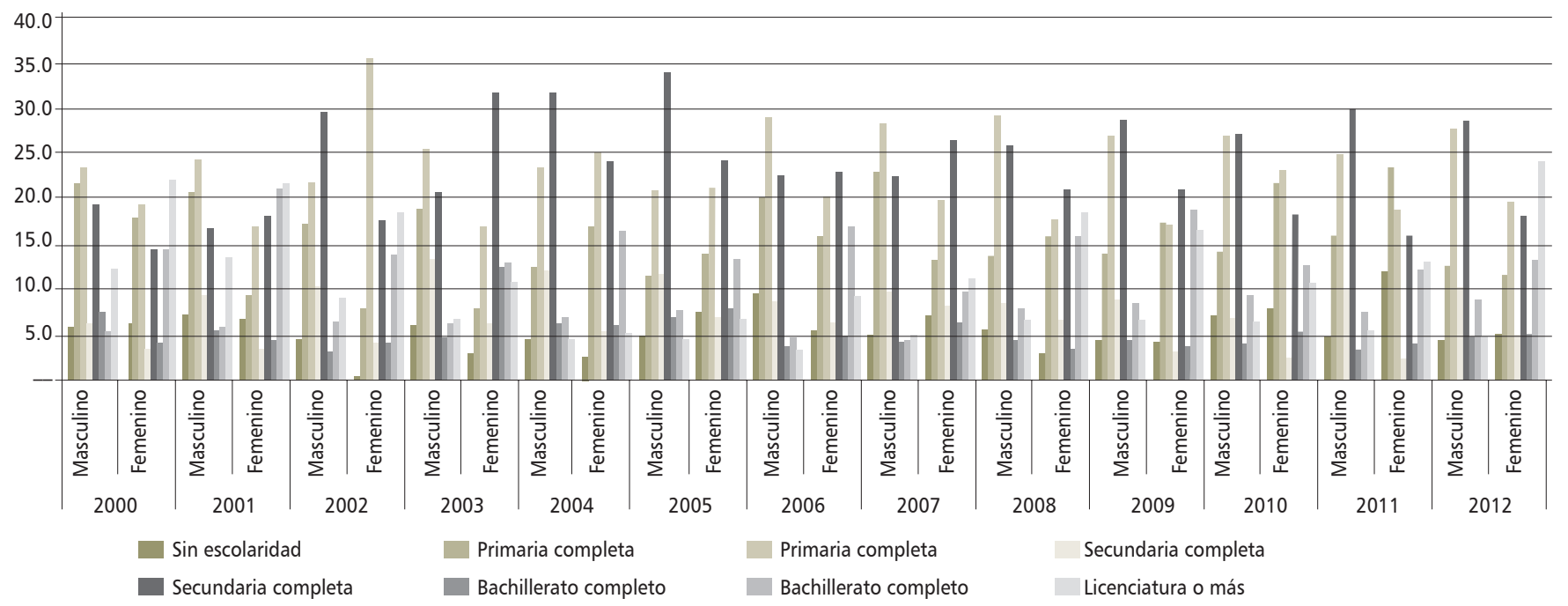

Fuente Elaboración propia, Encuesta sobre migración en la Frontera Norte de México, 2000-2012 
El análisis descriptivo de 12 años de la EMIF Norte (2000-2012) presenta información que se muestra en el tiempo las características específicas de cada grupo de población por sexo, el comportamiento de las categorías no es uniforme para los hombres ni para las mujeres, porque es una población heterogénea y su participación en la migración depende de factores estructurales como las crisis económicas tanto del país de origen como de destino, las reformas de políticas migratorias, pero también de proyectos individuales y familiares que responden a contextos comunitarios.

Sin duda, existe una migración femenina por motivos laborales que responde nichos laborales feminizados principalmente en el sector servicios, pero también a proyectos individuales y familiares como se ha demostrado en literatura que aludimos en otros apartados. La migración de los hombres sigue muy vinculada a la demanda de mano de obra del mercado laboral y es la principal afectada en las crisis económicas.

Se hace referencia a una población que proviene de localidades urbanas y no urbanas estas últimas posiblemente de estados como Veracruz y Chiapas en donde se ha deprimido la economía regional principalmente agrícola, obligando a la población que no tenía experiencia migratoria y redes a emigrar a Estados Unidos.

Pero también esta base presenta datos muy significativos respecto a la edad y escolaridad de la población migrante. Específicamente en el caso de las mujeres que tienen más de 40 años, es necesario conocer el ciclo de vida y familiar de estas mujeres que deciden dejar su lugar de origen. También llama la atención de la escolaridad tan alta de las mujeres respecto a los hombres, se parte del supuesto que son de procedencia urbana, profesionistas, que no necesariamente se están incorporando en trabajos acorde a su preparación.

Sin duda, el aumento del desempleo, el bajo poder adquisitivo del salario y la reducción en la calidad de vida de la población, pueden ser factores que ayuden a explicar la emigración de hombres y mujeres en México.
Lo que demuestra la información de la EMIF es que las características de los hombres y de las mujeres son diferentes, e inclusive existe diversidad en la población masculina y femenina a través del tiempo. Se hace referencia a una población heterogénea que requiere ser estudiada con mayor profundidad.

\section{Bibliografía}

- Alonso Meneses Guillermo (2012). "Los riesgos y estrategias del cruce clandestino de la frontera México-Estados Unidos desde un enfoque de género y familia: algunos datos y consideraciones para el periodo 1993-2010. En Migración y familia. Una mirada más humana para el estudio de la migración internacional. Salvador Berumen, Nina Frías y Julio Santiago (coordinadores) México, SPMAR-UPMCEM, INM Colección Migración.

- Alonso Meneses Guillermo (2012). "Rastros femeninos en el cruce clandestino de la frontera México-Estados Unidos. Algunos hechos, datos y sugerencias". En Género y Migración. Coordinadoras Esperanza Tunón Pablos, Martha Luz Rojas Wiesner. México, ECOSUR, El Colegio de Michoacán, El Colegio de la Frontera Norte, CIESAS. Volumen II.

- Arias Patricia y Ofelia Woo (2004). "Migración de la Zona Metropolitana de Guadalajara hacia Estados Unidos”, en Papeles de Población, vol 10. núm. 42, octubre-diciembre p 37-72.

- Ávila José Luis, Carlos Fuentes, Rodolfo Tuirán (2000), "Mujeres mexicanas en la migración a Estados Unidos”, en Migración México-Estados Unidos, continuidad y cambio. Rodolfo Tuirán (Coordinador), México, CONAPO, p 150-172 http://www.conapo. gob.mx/es/CONAPO/Migracion_Mexico-Estados Unidos_Continuidad_y_cambio consultado $24 \mathrm{de}$ febrero de 2005.

- Berumen Sandoval Salvador y Julio Santiago Hernández (2012), "Las mujeres en el proceso migratorio México-Estados Unidos. ¿Hacia una feminización 
de la migración?, en Migración y familia. Una mirada más humana para el estudio de la migración internacional. Salvador Berumen, Nina Frías y Julio Santiago (coordinadores) México, SPMAR-UPM-CEM, INM Colección Migración.

- Bustamante Jorge (1988), Migración de indocumentados de México a Estados Unidos. México, Fundación Friedrich Ebert Stiftung.

- Calva José Luis y Cesar Armando Salazar (2012). "Empleo digno y crecimiento económico". En Empleo digno, distribución del ingreso y bienestar. Análisis estratégico para el desarrollo, (Coordinador) José Luis Calva, México, Juan Pablos Editores, Volumen 11.

- CONAPO (2000), "Mujeres en la migración hacia Estados Unidos". Boletín Migración Internacional, año 5, número 13. http://www.conapo.gob.mx/work/ models/CONAPO/migracion_internacional/Boletines/bol16.pdf Consultado el 10 de octubre de 2005.

- CONAPO (2013). "La migración femenina mexicana a Estados Unidos. Tendencias actuales". Boletín de Migración Internacional, año 1, número 1. http:// www.conapo.gob.mx/work/models/OMI/Resource/652/1/images/boletinMigracionNo1 $8 \quad 03 \quad 13$. pdf consultado 09/03/2013

- Corona Vázquez Rodolfo (1988). "Modificaciones de las características del flujo migratorio laboral en México a Estados Unidos", en Migración y fronteras, (Coordinadores) Manuel Angel Castillo, El Colegio de la Frontera Norte/Asociación Latinoamericana de Sociología/ El Colegio de México, México.

- Corona Vázquez, Rodolfo (1995). "Modificaciones de las características del flujo migratorio laboral de México a Estados Unidos”, XX Congreso de la Asociación Latinoamericana de Sociología, México, D.F.

- Damían Araceli (2012). "Pobreza, crisis y política social en México". En Empleo digno, distribución del ingreso y bienestar. Análisis estratégico para el desarrollo, (Coordinador) José Luis Calva, México, Juan Pablos Editores, Volumen 11.

- Durand Jorge (1994). Más allá de la línea. Patrones migratorios entre México y Estados Unidos, México, Conaculta, Colección Regiones.

- Durand Jorge y Douglas Massey (2003). Clandestinos, México-Estados Unidos en los albores del siglo XXI, Universidad Autónoma de Zacatecas y Miguel Ángel Porrúa.

- Escobar Latapí Agustín, (1999). Utilidad, potencial y limitaciones de la Encuesta sobre Migración en la Frontera Norte de México, EMIF, desde la perspectiva de un antropólogo interesado en la migración". Comentarios a la Encuesta sobre Migración en la Frontera Norte de México, EMIF, Boletín, Migración Internacional, CONAPO, Año 3, núm 9 http://www. conapo.gob.mx/work/models/CONAPO/migracion internacional/Boletines/bol09.pdf consultado 11 de junio de 2013.

- Encuesta sobre migración en la frontera norte de México. Serie anualizada 2003-2010, SEGOB, CONAPO, INM, STPS, SER, COLEF.

- Gamio Manuel (1930). Quantitative Estimate, Source and Distribution of Mexican Immigration into the United States. México, Ed. Talleres gráficos.

- Giorguli Saucedo Silvia E, Selene Gaspar Olvera y Paula Leite (2007), La migración mexicana y el mercado de trabajo estadounidense. Tendencias, perspectivas y ioportunidades? México, SEGOB-CONAPO.

- Grieco Elizabeth; Boyd Monica (2003). "Women and Migration: Incorporating Gender into Internacional Migration Theory". Center for the Study of Population, Florida State University, College of Social Sciences. Working Paper

- Hondagneu-Sotelo Pierrete (1994). Gender Transition. Mexican Experiences of Immigration. University California Press.

- Marroni, Gloria (2005). "Daños Colaterales. La deportación de mexicanas desde Estados Unidos". Emigrantes Latinoamericanos, fronteras e identidades sociales. México, UNAM

- Pessar, Patricia (1999). "Engendering Migration Studies. The Case of New Immigrants in the United 
Status". American Behavioral Scientist, v. 42, n. 4,

- ...... (2007). "Mujeres, género y migración internacional en y más allá de las Américas: desigualdades y empoderamiento limitado", en Migración Internacional y desarrollo en América Latina y el Caribe. Editores, Paula Leite, Susana Zamora y Luis Acevedo. México, SEGOB- CONAPO.

- Portes Alejandro (1995). En torno a la informalidad: ensayos sobre teoría y medición de la economía regulada. México, Ed. Porrua y FLACSO

- Sassen-Koob Saskia y Smith Robert (1992). "Post-industrial Growth and Economic Reorganization: Their Impact Employment". In U.S.-Mexico Relations: Labor Market Interdependence. Jorge Bustamente, Clark W. Reynolds and Raúl Hinojosa, Stanford University Press.

- Trigueros Legarreta Paz, (1999). "Encuesta sobre Migración en la Frontera Norte de México (EMIF)”. En Comentarios a la Encuesta sobre Migración en la Frontera Norte de México, EMIF, Boletín, Migración Internacional, CONAPO, Año 3, núm 9 http://www. conapo.gob.mx/work/models/CONAPO/migracion
internacional/Boletines/bol09.pdf consultado $11 \mathrm{de}$ junio de 2013.

- Woo Morales Ofelia (1995), "Las mujeres mexicanas indocumentadas en la migración internacional y la movilidad transfronteriza”, en Mujeres, migración y maquila en la frontera norte. (Compiladoras) Soledad González, Olivia Ruíz, Laura Velasco y Ofelia Woo. México, El Colegio de México.

- ...-. (2001). Las mujeres también nos vamos al norte. México, Universidad de Guadalajara.

- ...-.(2004). Violencia contra la mujer migrante. Abuso y violencia a las mujeres migrantes. En Violencia contra la mujer en México. (Coordinadora) Teresa Fernández de Juan, México, Comisión Nacional de los Derechos Humanos, México.

- ....- (2007). "La experiencia migratoria de las mujeres urbanas hacia "El Norte", en ¿Campo o ciudad?". Nuevos espacios y formas de vida. (Coordinadoras) Patricia Arias y Ofelia Woo Morales, Guadalajara, Universidad de Guadalajara. 